\title{
Chronic Osteomyelitis following Gunshot Wounds
}

\author{
Maj WR Henderson \\ Maj LW Lauste
}

\section{INTRODUCTION}

This is an abridged version of the paper written by the late $W R$ Henderson and $L W$ Lauste in November/December 1944 while working at the Camp Hospital, Stalag 344 Lamsdorf, Silesia, Germany, based on their experience over the preceding four years. The paper was not offered for publication and discovered nearly fifty years later among $\overrightarrow{\mathrm{D}}$ memorabilia recording work done in a hospital for Allied Prisoners of War at Stadtroda, Thuringia, now in the Library of the Royal College of Surgeons of England. The full text is available from the Librarian at the College.

Both authors were captured in separate incidents by the Germans in May 1940 and remained in Germany until the end of: the war. Henderson, surgeon in charge of the Head Injury Unit was subsequently Senior British Medical Officer af్ Obermassfeld, the main hospital for British prisoners of war in central Germany. Lauste, surgical specialist at the 21s General Hospital, was later Senior British Medical Officer at Stadtroda. Both were moved by the Abwehr (security) in March 1943, Henderson to Colditz and Lauste to Treysa but in 1944 met at Lamsdorf where this paper was written.

The purpose of this paper is to discuss the treatment of chronic osteomyelitis following gunshot wounds as seen from about six months to two years after wounding. The term chronic osteomyelitis is used to indicate any persistent suppuration in connection with wounds of bone; it is thus used in a clinical and not in a pathological sense.

We have had occasion to treat a number of cases of longstanding chronic osteomyelitis in which there had been ample time for healing to occur. In these cases the underlying pathological causes of the persistent suppuration had become more evident with the passage of time, and it became apparent that when the cause could be removed healing occurred.

When healing did not occur it was usually due to incomplete removal of the cause owing to an inadequate operation, and further operations were required. We became more and more impressed with the importance of dealing thoroughly with the condition at the first exploration and the frequent necessity for an extensive procedure. Experience with more recent cases has shown there is nothing to be gained by delay in treatment and chronic sinuses should be dealt with radically as soon as the fracture is firmly united. Further, the severity of the condition and even its occurrence, may be reduced by minor procedures while the fracture is uniting.

The pathology of chronic osteomyelitis is discussed with special reference to the principles underlying treatment, and then the treatment of the condition, particularly those aspects of it which we feel have been insufficiently stressed.

\section{Pathology}

Operative findings have shown there are three main causes of non healing in long continued osteomyelitis. These are retained dead matter, persistent cavities in the bone and chronic osteitis.

\section{1) Retained Dead Matter}

This includes sequestra which are by far the commonest, metallic fragments and occasionally otheri materials such as clothing or debris of various kinds.

There follows a detailed discussion of sequestra ans their formation and of ring sequestra. The conclusion that the majority of sequestra arise from fragments bone completely or partly detached at the time of injury $\frac{\mathrm{P}}{\mathrm{D}}$

\section{2) Persistent Cavity in the Bone}

It is often found that sequestra lie in a cavity in the bone. Removal of the sequestra is sometimes followed $y^{s}$ permanent healing but, more often, healing does not ocegtip or is only temporary. Healing in these cases seems to 7 s determined chiefly by the size of the cavity, as a smalt cavity frequently heals while a large one rarely does so.

Healing is by the cavity being filled with fibrous tissu巴 and not by bone, and generally this is not possible i $\overrightarrow{F_{0}}$ large cavities.

\section{3) Chronic Osteitis}

In some cases a chronic osteitis is found to be the cause of the suppuration. In this condition there is a persisten infection of the surface layers of the bone which has a unhealthy wormeaten appearance, is soft and shows smale necrotic areas often with tiny sandlike sequestra.

This is discussed in some detail.

Finally, these three conditions may be found combined For instance, there may be areas of osteitis in the wall of cavity containing sequestra.

\section{The Infection}

The infection in chronic osteomyelitis is not due to specific organism, but is a mixed pyogenic infection. It i not the nature of the infection which is responsible foe maintaining suppuration but factors which allow th infection to persist. When these factors are removed the infection is readily overcome by the resistance of the body. 


\section{Healing in Bone}

Having enumerated the causes of chronic osteomyelitis it is of interest to consider how they operate and how healing takes place in the area after their removal. This problem is related to the union of the fracture and to healing in soft tissue wounds.

Then follows a long discussion of healing which takes place by fibrous tissue and the importance of vascular supply and allowing tissues to fall in naturally to fill any cavity.

\section{Permanence of Healing}

It is a clinical fact that many compound fractures, associated with varying degress of sepsis heal in the course of a few months and give rise to no further trouble. In these cases the infection has been overcome and the causes of chronic osteomyelitis as outlined above are either absent or have been removed at an early date. In other cases healing does not occur or is only temporary a matter of a few weeks. These come under the heading of chronic osteomyelitis as discussed in this article. In these cases if the cause can be removed completely, healing takes place, and experience has shown the wound remains healed.

Permanent healing cannot be assessed in any individual case since it is an accepted fact that osteomyelitis, healed and apparently cured, may at any time during the remainder of the patient's life again be the seat of an acute inflammatory process taking the form usually described as a flare. Clinically, however, a case which remains healed for several months may be regarded as permanently healed, though presumably a flare may occur later. To be clearly differentiated from this is the group in which healing is only temporary, usually a matter of weeks, and in which one of the causes of chronic osteomyelitis is still present and requires removal.

\section{The Flare}

An acute inflammatory process described as a flare may occur at any time in connection with an apparently permanently healed osteomyelitis. This condition is accepted as being due to the reactivation of organisms lying dormant in the tissues.

Then follows discussion of the flare and it is suggested that dead matter associated with latent infection is the cause.

\section{Treatment}

It follows from consideration of the pathology of chronic osteomyelitis that the treatment must be directed to removal of the cause of the persistent discharge and to provide suitable conditions to allow healing. These conditions are:

1) To provide and maintain free drainage.

2) To promote the obliteration of any space by healthy tissue during the process of healing. The underlying principles for the treatment of this condition are thus simple but in practice difficulties are frequently encountered. These difficulties are essentially anatomical in nature.
In the group of cases under discussion the fracture is united and the indication for operation is persistent or recurrent suppuration. The indication is thus clinical and not based on X-ray findings.

The time for operation is when the infection is quiescent as judged by the absence of signs of acute inflammation in the surrounding soft tissues, although occasionally it may be necessary to operate in the subacute stages when the operation should be modified to a minimum

\section{$X$-Ray Examination}

In addition to the information obtained by clinical examination an X-ray, although not essential, is highly desirable. It is important to realise the value and the limitations of X-ray findings.

The various difficulties in interpreting $X$-rays are discussed.

To sum up, an X-ray often fails to show the cause and extent of the inflammatory process and repeated X-rays after an interval of time rarely give any further information. The main value of the $\mathrm{X}$-ray is to show the configuration of the healed fracture, the sites of strong union and which portions of bone can with safety and to the best advantage be removed in the obliteration of cavities.

\section{Lipiodol Injection}

An X-ray after injection of a sinus with lipiodo through an opaque catheter may give additionat information. This is not necessary in the limbs, but mayo be of great help in regions such as the pelvis. Here the focus of the disease is not always certain and there mayo be a long sinus which enters the pelvis or extends across the midline. This information will also help to plan the best approach which is not necessarily along the sinus.

\section{Bacteriological Examination}

We have not made routine bacteriological examinations of the pus as this does not appear to be of any practical value except in rare cases in which diphtheritic infection is suspected.

After some general discussion the types of operation required are discussed under four main headings. These are Simple sequestrectomy or removal of foreign body, The Extensive operation, Recurrent or persistent inflammation and The Flare.

\section{After Treatment}

The two methods of treatment commonly employed after operation are the closed plaster method and ordinary splintage with frequent dressings. We have used both but as a rule prefer the latter.

The advantage of the closed plaster method is economy of time and material, and selected cases progress well with this treatment. The disadvantages are that it is not always possible to obtain the completely open drainage required, and periodic examination of the wound is important to be sure drainage remains adequate and progress is satisfactory. Also complete immobilisation is 
not required as there is no fracture and no fresh acute infection in the soft tissues.

Our usual practice is to use frequent dressings as convalescence is smoother, the patients are more comfortable and pocketing is recognised quickly. The closed plaster we use sometimes in cases when there is no fear of pocketing or the fracture line is unduly weak.

Then follows detailed discussion under various headings and the importance of examination during healing.

Experience has shown that after operation it is of great value to explore the wound under anaesthesia in about three weeks. By this means it can be ascertained that healing is progressing well throughout the wound.

\section{Accessory Methods to Promote Healing}

In the past it has been recommended that the dead space in cavities should be obliterated by introducing substances such as wax and fat. We have no experience of this method but do not believe the infection can be reduced sufficiently to prevent these dead materials acting as a foreign body in which case one of the main causes of osteomyelitis is reproduced.

The introduction of living tissue in the form of a muscle pedicle into the cavity has been advocated. It is difficult to get the muscle to remain without fixation and there is danger of abscess formation between the wall of the cavity and the muscle, especially if this is done before a healthy granulating surface has been obtained. Our small experience has indicated that it is a difficult and unsatisfactory method. We prefer the usual method in which intact muscle gradually becomes approximated to the surface of the bevelled cavity and there is free drainage during the process. Furthermore, muscle grafts are not readily available in some situations.

The transplantation of living tissues has a limited application in chronic osteomyelitis, but a skin graft can sometimes be used with advantage in the final healing of the subcutaneous surface of the tibia.

\section{Regional Difficulties}

Before discussing regional difficulties two facts of general importance are worthy of mention.

Chronic osteomyelitis is more common in the lower than in the upper limb and more difficult to cure. There are several reasons for this. The depth of the bone hinders drainage through the thick layer of muscles and strong inter-muscular septa; this is particularly seen in the thigh. The natural and optimum drainage in the limb is through the extensor surfaces which are more superficial and surgically more accessible, but in the usual supine position of nursing such drainage is against gravity in the lower limb; this is well shown in the tibia. The large size of the lower limb bones predisposes to the formation of large cavities. The converse is found in the upper limb where the bones are smaller, more superficial and more favourably placed for dependent drainage through the extensor surfaces.

Healing is more difficult to secure in cases which are very chronic or have had many operations. The sclerosis of bone and increased fibrosis of soft tissues commonlye?

found are largely responsible for this.
There is an account of problems involving long bones and the special difficulties in the foot and pelvis.

\section{Treatment of Early Gunshot Wounds of Bone}

Lessons learned in the treatment of chroni osteomyelitis have impressed upon us the importance of removing all sequestra at an early stage as is stressed in present day teaching on the treatment of war wounds. If has been noticed that after the early removal of sequestrat a cavity in the bone may heal spontaneously, either bs new bone formation or fibrous tissue. A cavity of similae size in chronic osteomyelitis would require collapsing t⿳亠े, heal. It therefore appears that in the early stages, if ther is no cause to maintain a cavity, healing can take place, We believe this to be due to the greatly increased vascularity of the region in the early stages which facilitates healing, while in chronic cases the increased fibrosis reduced blood supply to an extent that healieg does not occur.

If such is the case it is of even greater importancestos remove sequestra as early as possible. Owing to difficulty of recognising them by X-ray all dischargeng wounds should be explored periodically while the fracturr $\overrightarrow{\varepsilon_{0}}$ is uniting. Such operations are limited to explorationgुg the wound with a finger in order to identify and remsie loose sequestra without disturbing healthy tissue or healing fracture.

\section{Summary}

There are three main causes, often combined, of chronic osteomyelitis due to gunshot wounds. These are retained dead matter, cavities and osteitis and are ofte combined. If these causes are removed the infection subsides and healing can occur. The indication for operation is clinical - persistent suppuration - and not the $\mathrm{X}$-ray appearances. The nature and extent of the operation can only be determined when the sinus is explored an the cause of the suppuration ascertained. The principles of the operation are:

1) To remove the cause.

2) To provide free drainage.

3) To provide suitable mechanical condition for healing i.e. to facilitate the obliteration of resultant spaces. Whe these principles can be satisfactorily applied healing occurs, but the difficulty or impossibility of applying them in some cases results in failure to cure the condition These difficulties are anatomical. Early removal of sequestra is of great importance in reducing the severity of chronic osteomyelitis and may even prevent it. 